\title{
QoS-Oriented Dynamic Power Allocation in NOMA-based Wireless Caching Networks
}

\author{
Yue Yin, Student Member, IEEE, Miao Liu, Member, IEEE, and Guan Gui, Senior Member, IEEE, \\ Haris Gacanin, Senior Member, IEEE, and Fumiyuki Adachi, Life Fellow, IEEE
}

\begin{abstract}
Non-orthogonal multiple access (NOMA) based wireless caching network (WCN) is considered as one of the most promising technologies for next-generation wireless communications since it can significantly improve the spectral efficiency. In this paper, we propose a quality of service $(\mathrm{QoS})$-oriented dynamic power allocation strategy for NOMA-WCN. In content stack phase, the base station sends multiple files to the content servers by allocating different powers according to the different QoS targets of files, for ensuring that all content servers can successfully decode the two most popular files. In content deliver phase, the content servers serve two users at the same time by allocating the minimum power to the far user according to the QoS requirement, and then all the remaining power is allocated to the near user. Hence, the proposed power allocation scheme is able to increase the hit probability and drop the outage probability compared with conventional method. Simulation results confirm that the proposed power allocation method can significantly improve the caching hit probability and reduce the user outage probability. It is also shown that this strategy can reduce the user delay time, improve the system efficiency and the capacity.
\end{abstract}

Index Terms-Non-orthogonal multiple access, wireless caching networks, dynamic power allocation, quality of service.

\section{INTRODUCTION}

Spectral efficiency is one of the critical design challenges in the sixth generation (6G) wireless communication systems [1]-[3]. To improve the spectral efficiency, wireless caching techniques have been proposed to divide traffic load at the base station into many content servers [4]-[6]. In a typical communication system, the base station accesses the core network to download the file and then transmits it to users. On the other hand, the main idea of wireless caching is to download the popular content by the content servers during the content stack phase before it is requested. Consequently the users can be served locally. Some researchers studied the content placement of wireless caching, for example, S. H.

This work was supported by the Project Funded by the National Science and Technology Major Project of the Ministry of Science and Technology of China under Grant TC190A3WZ-2, the Jiangsu Specially Appointed Professor under Grant RK002STP16001, the Innovation and Entrepreneurship of Jiangsu High-level Talent under Grant CZ0010617002, the Six Top Talents Program of Jiangsu under Grant XYDXX-010, the 1311 Talent Plan of Nanjing University of Posts and Telecommunications. (Corresponding author: Guan Gui)

Y. Yin, M. Liu and G. Gui are with the College of Telecommunications and Information Engineering, Nanjing University of Posts and Telecommunications, Nanjing 210003, China (E-mails: $\{1018010407$, liumiao, guiguan $\} @$ njupt.edu.cn)

H. Gacanin is with Nokia Bell Labs, 2018 Antwerp, Belgium (e-mail: harisg@ieee.org)

F. Adachi is the Research Organization of Electrical Communication, Tohoku University, Sendai 980-8577 Japan (e-mail: adachi@ecei.tohoku.ac.jp)
Chae, et al. [7] control cache-based channel selection diversity with sophisticated considerations of wireless fading channels and interactions among multiple users. J. Kwak, et al. [8] proposed a hybrid content caching scheme design that does not require the knowledge of content popularity. They optimize the content caching locations by employing the Lyapunov optimization approach. To decrease the latency and improve the utilization of caches, C. Liang, et al. [9] designed a mechanism to jointly provide proactive caching, bandwidth provisioning, and adaptive video streaming.

Non-orthogonal multiple access (NOMA) has been recognized as one of the most promising wireless technologies for improving the spectrum efficiency in 6G mobile communications [10]. NOMA enables different users to occupy the same spectrum, time, space through power multiplexing, further improves spectrum utilization and reduces users' time delay [11]-[14]. At the receiver, the signals of multiple users are separated by the successive interference cancellation (SIC). User grouping and power allocation are the two most important parts of the NOMA system. For user grouping, Y. Yin et al. proposed a dynamic user grouping strategy to decrease the bit error rate [15]. Z. Ding, et al. [16] firstly introduced NOMA into $\mathrm{WCN}$, where a fixed power allocation in the caching deliver phase, thus the allocated power might be unable to satisfy the users' QoS demands dynamically. Z. Zhao, et al. [17] studied the coverage performance of NOMA-WCN while did not consider about power allocation scheme. To solve this problem, Y. Fu, et al. [18] studied the dynamic power allocation driven by deep neural network of WCN based on NOMA. However, this method requires a lot of data training while it is hard to obtain in the realistic systems.

In this paper, we propose a dynamic power allocation strategy for NOMA-WCN based on the QoS orientations of both files and users. A closed form solution of QoSoriented power allocation is derived to reduce deployment costs for both base stations and content servers. Hence, our proposed QoS-oriented dynamic power allocation method can be utilized in a realistic NOMA-WCN. Computer simulations are conducted to evaluate the proposed method with respect to hit probability and the outage probability illustrating the effectiveness of the proposed method.

\section{Problem Formulation}

The transmission of WCN is divided in the two independent time phases, namely content stack phase and content deliver phase as shown in Fig. 1. Firstly, the base station predicts 
which files are popular for users in the next period, and then downloads these files from the core network. During the content stack phase, the base station transmits these files to all content servers. At this phase, the base station and the content servers are unable to serve the users, resulting in increasing their time delays. Therefore, the content stack phase should be short. However, if the content stack phase is too short, content servers will not have enough time to download enough files, so we apply NOMA to handle this problem. Then in the content deliver phase, when the users request files from the content servers, if the files exist in their corresponding content servers, the content servers will directly send the files to users. If the content server does not stack the files which are requested by users in advance, the base station will serve users directly. However, base station will increase the load of the backhaul link and cause the network congestion. This case is not considered in this paper.

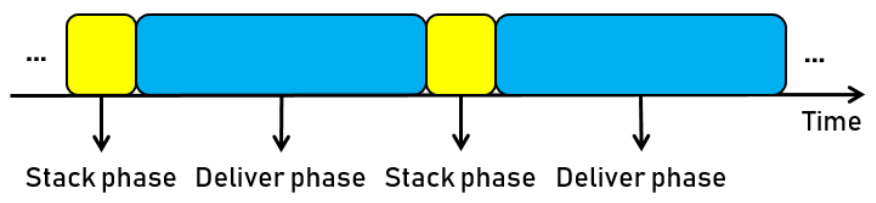

Fig. 1. Time-phase diagram of content stack and content deliver in a WCN The content stack phase and the content deliver phase are independent of each other. During the content stack phase, the content server cannot serve users. This period is set short to avoid increasing user time delay.

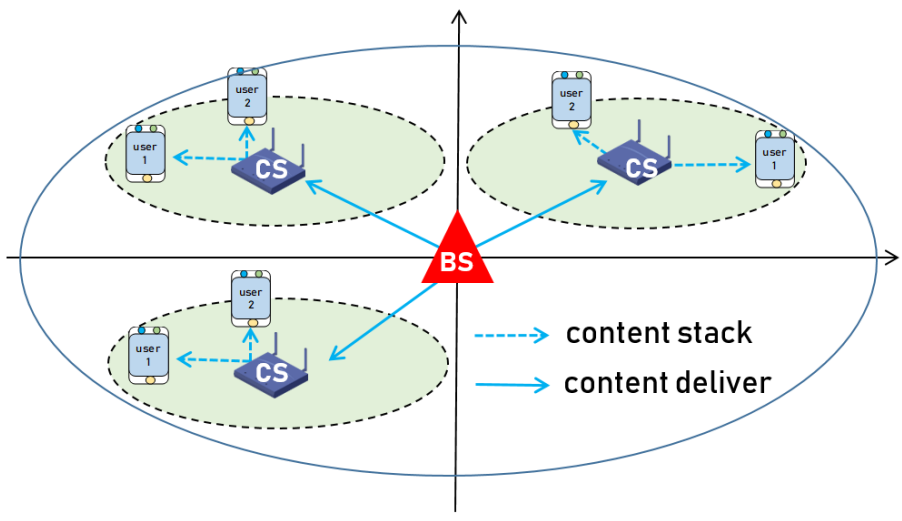

Fig. 2. System model of the WCN, BS refers to the base station, CS refers to the content server. There are multiple content servers within the coverage area of the base station. Each content server serves multiple users.

We consider $C$ content servers distributed within the coverage of a base station, and each content server serves multiple mobile users within its deliver range, as shown in Fig. 2. Moreover, it is assumed that each user is only associated with the nearest content server. In the system model, the position of the base station is set to the origin of the plane coordinates, and the content servers follow a homogeneous Poisson distribution with the base station as the center. The popularity of the files are modeled by the Zipf distribution [19]. Zipf's law has proved that if the words in the dictionary are sorted according to the frequency of their occurrence, the frequency of each word is inversely proportional to its order number,

$$
G(r)=\frac{A}{r^{\varepsilon}},
$$

where $r$ represents the frequency order of the words. $G(r)$ is the frequency of occurrence of the $r$-th word. $A$ and $\varepsilon$ are constants, where $A$ is approximately equal to 0.1 and $\varepsilon$ is the popularity parameter. Assuming the total number of all the requested files is $F_{\text {total }}$, thus the popularity of file $f$ is computed as:

$$
G(f)=\frac{\frac{1}{f^{\varepsilon}}}{\sum_{i=1}^{F_{\text {total }}} \frac{1}{i^{\varepsilon}}}
$$

The larger the value of $\varepsilon$, the more popular the top-ranked files are.

\section{The Proposed QoS-Oriented Dynamic Power ALLOCATION ALGORITHM}

In this section, we present a QoS-oriented dynamic power allocation method in the content stack phase and the content deliver phase.

\section{A. In Content Stack Phase}

Base station predicts files which are the most popular ones in the next period, and downloads these files from the core network. During the content stacking phase, the base station send these files to the content servers. All files are allocated with different powers by the NOMA in the base station. Each file $f$ has QoS requirement $R_{f}^{\text {QoS }}$, and it can be decoded correctly on the receiver only when the QoS requirement is satisfied. If the file gets more power, it is more likely to be decoded correctly at the content servers. In addition, if the content server is close to the base station, channel conditions is better and it is easier to transmit the files successfully to the content servers. Therefore, the closer the content server is to the base station, the more files can be decoded correctly.

In this paper, we only consider large-scale fading channel, and the path loss channel model from $\mathrm{CS}_{l}$ to base station is:

$$
d_{l}=\frac{1}{\left(\sqrt{\left|x_{l}-x_{0}\right|^{2}+\left|y_{l}-y_{0}\right|^{2}}\right)^{3}},
$$

where $\mathrm{CS}_{l}$ 's position is $\left(x_{l}, y_{l}\right)$, the base station's position is $\left(x_{0}, y_{0}\right)$, and the path loss factor is 3 . In order to reduce the computational complexity, we assume that the base station is located at the origin of the two-dimensional plane, hence the path loss model of $\mathrm{CS}_{l}$ can be simplified as

$$
d_{l}=\frac{1}{\left({\sqrt{x_{l}^{2}+y_{l}^{2}}}^{3}\right.}
$$

The base station sends a superimposed signal of 3 popular files to content servers at one time slot. And it is expected that at least the two most popular files can be correctly decoded by all content servers. The rate for $\mathrm{CS}_{l}$ to decode the most popular file $f_{1}$ is:

$$
R_{l, 1}=\log _{2}\left(1+\frac{d_{l} P_{s} \alpha_{1}}{d_{l} P_{s}\left(\alpha_{2}+\alpha_{3}\right)+n_{0}}\right)
$$


where $P_{s}$ is the total power sent by the base station, $\alpha_{1}, \alpha_{2}$, and $\alpha_{3}$ are the power allocation factors of files $f_{1}, f_{2}$, and $f_{3}$, respectively, and $n_{0}$ is noise power.

After passing through the fading channel, the file with higher power is more likely to be decoded correctly, so power is allocated to the most popular file $f_{1}$ first. The QoS of file $f_{1}$ is $R_{1}^{\mathrm{QoS}}$. $\mathrm{CS}_{l}$ is able to successfully decode file $f_{1}$ only when $R_{l, 1} \geq R_{1}^{\mathrm{QoS}}$,

$$
\log _{2}\left(1+\frac{d_{l} P_{s} \alpha_{1}}{d_{l} P_{s}\left(\alpha_{2}+\alpha_{3}\right)+n_{0}}\right) \geq \log _{2}\left(1+S N R_{1}^{\mathrm{QoS}}\right)
$$

where $S N R_{1}^{\mathrm{QoS}}$ is SNR requirements for file $f_{1}$. The power allocation factors for all files satisfy $\sum_{j=1}^{3} \alpha_{j}=1$. As a result, the power allocation factor for file 1 must satisfy:

$$
\alpha_{1} \geq \frac{d_{l} P_{s} S N R_{1}^{\mathrm{QoS}}+n_{0} S N R_{1}^{\mathrm{QoS}}}{\left(S N R_{1}^{\mathrm{QoS}}+1\right) d_{l} P_{s}} .
$$

Then, $\alpha_{1}$ can be determined by:

$$
\alpha_{1}=\min \left\{1, \frac{d_{l} P_{s} S N R_{1}^{\mathrm{QoS}}+n_{0} S N R_{1}^{\mathrm{QoS}}}{\left(S N R_{1}^{\mathrm{QoS}}+1\right) d_{l} P_{s}}\right\} .
$$

Similarly, after perfect SIC, the interference power from file $f_{1}$ can be canceled, and then the data rate for $\mathrm{CS}_{l}$ to decode the second most popular file $f_{2}$ is:

$$
R_{l, 2}=\log _{2}\left(1+\frac{d_{l} P_{s} \alpha_{2}}{d_{l} P_{s} \alpha_{3}+n_{0}}\right) .
$$

Hence, the power allocation factor $\alpha_{2}$ of file $f_{2}$ can be further calculated as:

$\alpha_{2}=\min \left\{1-\alpha_{1}, \frac{d_{l} P_{s} S N R_{2}^{\mathrm{QoS}}\left(1-\alpha_{1}\right)+n_{0} S N R_{2}^{\mathrm{QoS}}}{\left(S N R_{2}^{\mathrm{QoS}}+1\right) d_{l} P_{s}}\right\}$.

Finally, file $f_{3}$ is decoded by the content server as far as possible. Thus the power allocation factor $\alpha_{3}$ can be taken as:

$$
\alpha_{3}=\max \left\{1-\alpha_{1}-\alpha_{2}, 0\right\} .
$$

\section{B. In Content Deliver Phase}

During the content deliver phase, the users request files from their content servers. By applying NOMA, the files requested by two users can be superimposed and transmitted on the same spectrum simultaneously. After receiving the signal, the user who is near the content server is able to use SIC for separating the useful signal. In detail, the superimposed signal received by the near user of $\mathrm{CS}_{l}$ is

$$
\begin{aligned}
y_{l, \text { near }} & =h_{l, \text { near }} \sqrt{d_{l, \text { near }} \beta_{l, \text { near }}} f_{l, \text { near }} \\
& +h_{l, \text { near }} \sqrt{d_{l, \text { near }} \beta_{l, \text { far }}} f_{l, \text { far }} \\
& +\sum_{q \in \Phi C \backslash l} h_{q, \text { lnear }} \sqrt{d_{q, \text { lnear }} \beta_{q, \text { near }}} f_{q, \text { near }} \\
& +\sum_{q \in \Phi C \backslash l} h_{q, \text { lnear }} \sqrt{d_{q, \text { lnear }} \beta_{q, \text { far }}} f_{q, \text { far }} \\
& +n_{l, \text { near }},
\end{aligned}
$$
Therefore, the power allocation factor $\beta_{l, f a r}$ for the far user
should satisfy

$$
\begin{aligned}
\beta_{l, f a r} & \geq \frac{S I N R_{f a r}^{Q o S} d_{l, f a r} P_{C}+S I N R_{f a r}^{Q o S} n_{0}}{\left(1+S N R_{f a r}^{Q o S}\right) d_{l, f a r} P_{C}} \\
& +\frac{S I N R_{f a r}^{Q o S} I_{\text {inter }} P_{C}}{\left(1+S N R_{f a r}^{Q o S}\right) d_{l, f a r} P_{C}} .
\end{aligned}
$$

where $\beta_{l, f a r}$ is the power of the far user of $\mathrm{CS}_{l}, d_{l, f a r}$ is the path loss of the far user of $\mathrm{CS}_{l}$, and $P_{C}$ is the transmit power of the content servers. Hence, $I_{\text {inter }}=$ $\sum_{q \in \Phi C \backslash l}\left|h_{q, l \text { far }}\right|^{2} d_{q, l \text { far }}$ which is the intergroup interference of NOMA, where $d_{q, l f a r}$ is the path loss of the far user of $\mathrm{CS}_{l}$ to $\mathrm{CS}_{q}$.

The far user can successfully decode the requested file when the rate is no less than its QoS $R_{f a r}^{\text {QoS }}$. That is

$$
\begin{array}{r}
\log _{2}\left(1+\frac{\beta_{l, \text { far }} d_{l, f a r} P_{C}}{\beta_{l, \text { near }} d_{l, f a r} P_{C}+I_{\text {inter }} P_{C}+n_{0}}\right) \\
\geq \log _{2}\left(1+S N R_{\text {far }}^{Q o S}\right) .
\end{array}
$$

Since $\beta_{l, f a r}+\beta_{l, \text { near }}=1$, we obtain

$$
\beta_{l, f a r}=\min \left\{\begin{array}{l}
1, \frac{S I N R_{f a r}^{Q o S} d_{l, f a r} P_{C}+S I N R_{f a r}^{Q o S} n_{l, f a r}}{\left(1+S N R_{f a r}^{Q O S}\right) d_{l, f a r} P_{C}} \\
+\frac{S I N R_{f a r}^{Q O S} I_{\text {inter }} P_{C}}{\left(1+S N R_{f a r}^{Q o S}\right) d_{l, f a r} P_{C}}
\end{array}\right\} .
$$

In addition, the power allocation factor of the near user is

$$
\beta_{l, \text { near }}=1-\beta_{l, f a r} .
$$

According to the aforementioned presention, the proposed QoS-oriented dynamic power allocation algorithm for NOMAWCN is summarized in Algorithm 1. 
$\overline{\text { Algorithm } 1 \text { The proposed QoS-oriented dynamic power }}$ allocation algorithm for NOMA-WCN.

Input: the total number of files $F_{\text {total }}$, the popularity parameter $\alpha$, the total power of base station $P_{s}$, the total power of the content servers $P_{c}$ and the QoS of files and users;

Output: The powers of files and users;

1: Base station generates the popularity of files $G(f)$ according to (2) and downloads the most popular three files;

2: Base station allocates the power of the most popular file according to (8);

3: Then, base station allocates the power of the second most popular file according to (10);

4: The remaining power is given to the third file;

5: Base station superimposes the three files together and sends them to the content servers;

6: The content servers allocate the power to the far users according to (16), and the remaining power is fully distributed to the near users;

\section{Simulation Results}

In this section, we evaluate the proposed power allocation method in NOMA-WCN during the content stack phase and content deliver phase separately. Firstly, we use the content hit probability and user outage probability to measure the performance of the proposed power allocation strategy during the content stack phase and the content delivery phase, respectively. The content hit probability is the probability that the content servers have the files, which the users request. Since the content hit probability is related to the file popularity and the file outage probability, we can express $\mathrm{CS}_{l}$ 's hit probability as

$$
H i t(l)=\sum_{f=1}^{3} G(f)\left(1-P_{l, f}\right)
$$

where $G(f)$ represents the popularity of file $f, P_{l, f}$ is the outage probability for $\mathrm{CS}_{l}$ to decode file $f$. When the rate of $\mathrm{CS}_{l}$ to decode the file $f$ is less than the file $f$ 's QoS requirement, the transmission of file $f$ is regarded as the outage.

In the content deliver phase, the user outage probability is the probability that the rate the user decodes the requested file is less than the QoS requirement. We set the coverage radius of the base station is 50 meters. The total number of files in the file library is 5 , and the popularity parameter $\varepsilon$ is 0.5 . The total number of content servers is 5 . We compare the strategy proposed in this paper with that proposed by Ding et al. [16]. During the content stack phase, Ding et al.'s power allocation method is to satisfy the most popular file being correctly decoded by all content servers. The power of the second file and third file is $3 / 4,1 / 4$ of the remaining power, respectively. In the content deliver phase, the power allocation in paper [16] is fixed. The power allocation factor of the near user is $1 / 4$, and the power allocation factor of the far user is $3 / 4$.

Fig. 3 shows the outage probability of the cache during the content stack phase. It can be seen from this figure that when the total power is $30 \mathrm{dBm}$, the outage probability of our proposed power allocation method is $28 \%$ and $15 \%$ lower than that of the method proposed by Z. Ding et al. [16]. In

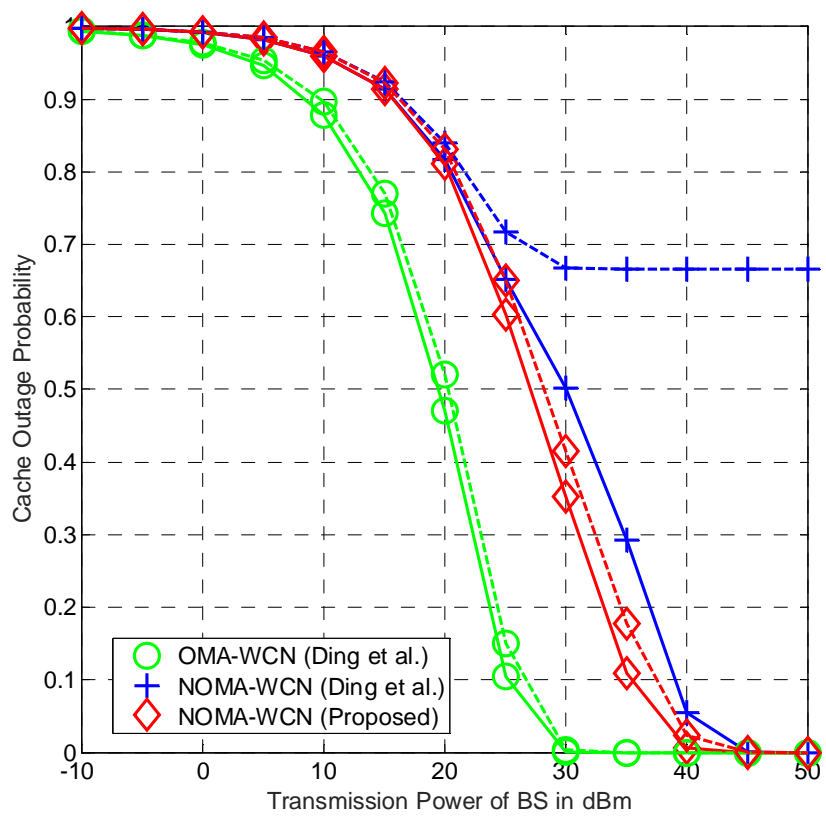

Fig. 3. Cache outage probability vs. transmit power of BS. The dotted line denotes the outage probability when the QoS of all three files is 2 bit per channel use (i.e., $R_{1}=R_{2}=R_{3}=2$ ). The solid line denotes the probability of outage when the QoS of all three files is 1.8 bit per channel use (i.e., $R_{1}=R_{2}=R_{3}=2$ ).

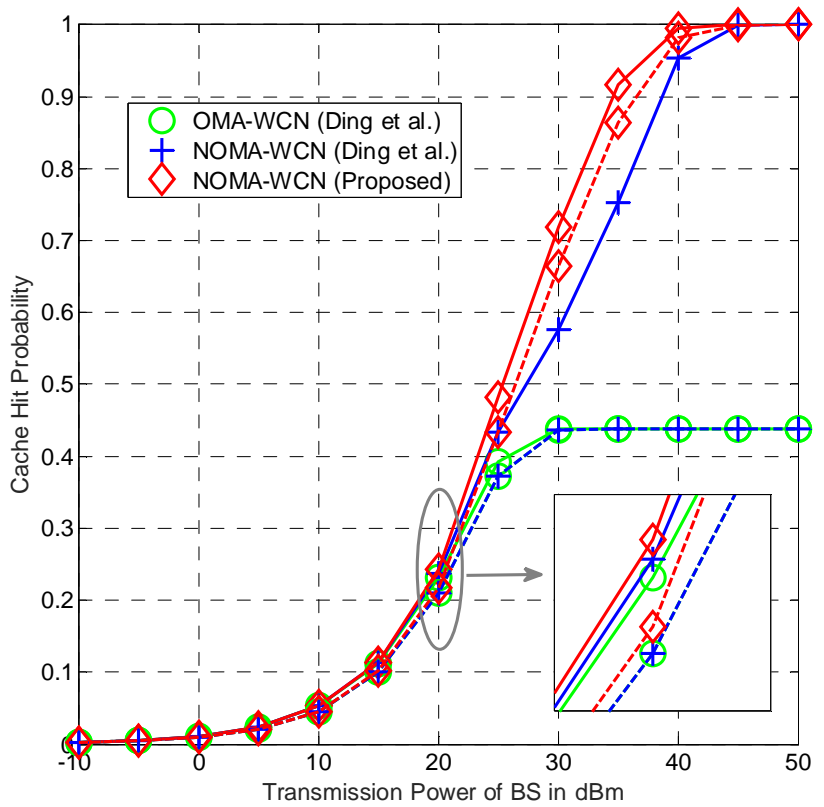

Fig. 4. Cache hit probability vs. transmit power of BS. The dotted line denotes the outage probability when the QoS of all three files is 2 bit per channel use (i.e., $R_{1}=R_{2}=R_{3}=2$ ). The solid line denotes the probability of outage when the QoS of all three files is 1.8 bit per channel use (i.e., $R_{1}=R_{2}=R_{3}=2$ ).

Fig. 4, the popularity parameter is 0.5 and the total number of file library files is 5 , and the cache hit probability is increased by about $20 \%$ and $28 \%$, respectively. Fig. 5 shows the outage probability of the near user and the far user during the cache deliver phase. The QoS requirements of the far user and the near user are 1 bit per channel use and 6 bit per channel use, respectively. When the total power is $15 \mathrm{dBm}$, the outage 
probability of the far user is reduced by $5 \%$ and the outage probability of the near user is reduced by about $10 \%$. From the simulation results, we can observe that the proposed method can effectively improve the cache hit probability of the WCN and reduce the probability of user outage compared with the state-of-the-art method [16]. Hence, our proposed QoSoriented dynamic power allocation method can be considered as a candidate technology to deploy in NOMA-WCN.

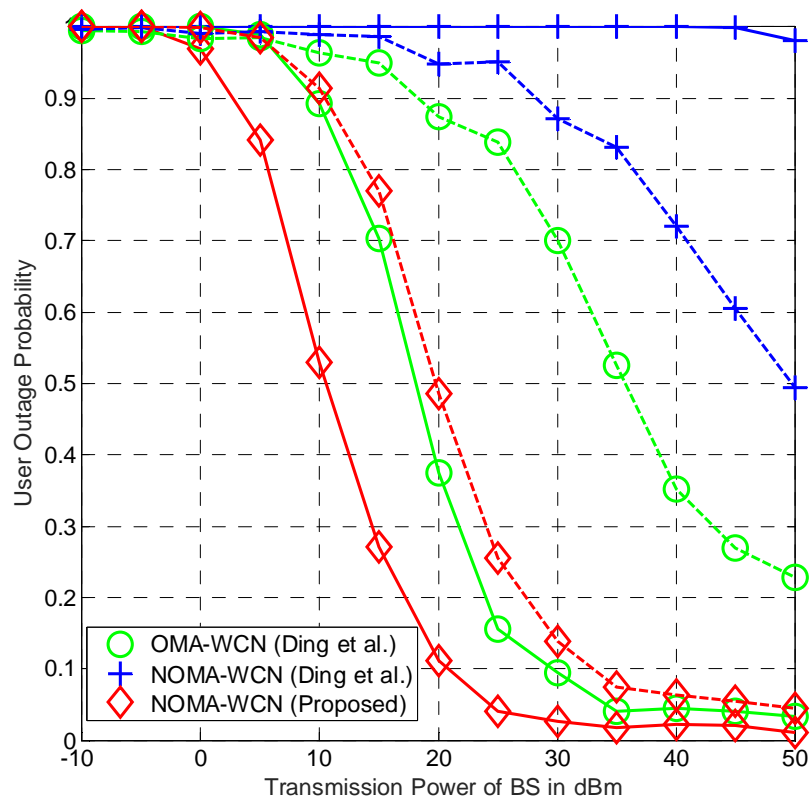

Fig. 5. User outage probability vs. transmit power. The QoS of far user is set as $2 \mathrm{bit} / \mathrm{channel}$ use and the QoS of near user is set as $7 \mathrm{bit} /$ channel use. The solid line denotes the probability of outage of the far user. The dashed line denotes the probability of outage of the near user.

\section{CONCLUSION}

In this paper, we have proposed a QoS-oriented power allocation strategy in content stack phase and content deliver phase for NOMA-WCN. In the content stack phase, the base station applies NOMA to assign different powers to multiple files for superimposition and transmission. The strategy of the power allocation in this phase is to ensure that the two most popular files can be correctly decoded by the all content servers. In the content deliver phase, content servers use NOMA technology to serve two users on the same spectrum at the same time. The goal of power allocation in this phase is to ensure that files of far users can be correctly decoded, reducing user delay and improving spectrum efficiency. Simulation results confirmed that the proposed power allocation strategy improves the cache hit probability and reduces the user outage probability compared with the OMA scheme and a fixed power allocation based NOMA scheme. In future work, we will consider researching the user grouping technology of NOMA in WCN to further increase the cache hit probability and reduce the user outage probability.

\section{ACKNOWLEDGEMENT}

We thank Prof. Zhiguo Ding from The University of Manchester in assistance with reproducing the power alloca- tion method for NOMA-WCN [16] and also many helpful discussions that greatly improved the manuscript.

\section{REFERENCES}

[1] F. Tang, Y. Kawamoto, N. Kato, and J. Liu, "Future intelligent and secure vehicular network towards 6G: Machine-learning approaches," Proc. IEEE, vol. 108, no. 2, pp. 292-307, Feb. 2020.

[2] Z. Shi, W. Gao, S. Zhang, J. Liu, N. Kato, "AI-enhanced cooperative spectrum sensing for non-orthogonal multiple access," IEEE Wireless Commun. Mag., in press, doi: 10.1109/MNET.001.1900305

[3] N. Kato, B. Mao, F. Tang, Y. Kawamoto, and J. Liu, "Ten challenges in advancing machine learning technologies towards 6G," IEEE Wireless Commun. Mag., in press, doi: 10.1109/MNET.001.1900476

[4] C. Yang, B. Xia, W. Xie, K. Huang, Y. Yao, and Y. Zhao, "Interference cancelation at receivers in cache-enabled wireless networks," IEEE Trans. Veh. Technol., vol. 67, no. 1, pp. 842-846, Jan. 2018.

[5] F. Cheng, G. Gui, N. Zhao, Y. Chen, J. Tang, and H. Sari, "UAV-relayingassisted secure transmission with caching," IEEE Trans. Commun., vol. 67, no. 5, pp. 3140-3153, May 2019.

[6] N. Zhao, F. Cheng, F. Yu, J. Tang, Yu. Chen, G. Gui, and H. Sari, "Caching UAV assisted secure transmission in hyper-dense networks based on interference alignment," IEEE Trans. Commun., pp. 22812294, vol. 66, no. 5, May 2018.

[7] S. H. Chae, and W. Choi, "Caching placement in stochastic wireless caching helper networks: Channel selection diversity via caching," IEEE Trans. Wireless Commun., vol. 15, no. 10, pp. 6626-6637, Oct. 2016.

[8] J. Kwak, Y. Kim, L. B. Le, and S. Chong, "Hybrid content caching in 5G wireless networks: Cloud versus edge caching," IEEE Trans. Wireless Commun., vol. 17, no. 5, pp. 3030-3045, May 2018.

[9] C. Liang, Y. He, F. R. Yu, and N. Zhao, "Enhancing QoE-aware wireless edge caching with software-defined wireless networks," IEEE Trans. Wireless Commun., vol. 16, no. 10, pp. 6912-6925, Oct. 2017.

[10] G. Gui, M. Liu, F. Tang, N. Kato, and F. Adachi, "6G: Opening new horizons for integration of comfort, security and intelligence," IEEE Wireless Commun. Mag., in press, doi: 10.1109/MWC.001.1900516.

[11] Y. Saito, Y. Kishiyama, A. Benjebbour, T. Nakamura, A. Li, and K. Higuchi, "Non-oOrthogonal multiple access (NOMA) for cellular future radio access," Proc. VTC 2013-Spring, Dresden, Germany, 2-5 June 2013, pp. $1-5$.

[12] Z. Ding, Z. Yang, P. Fan, and H. V. Poor, "On the performance of non-orthogonal multiple access in $5 \mathrm{G}$ systems with randomly deployed users," IEEE Signal Process. Lett., vol. 21, no. 12, pp. 1501-1505, Dec. 2014.

[13] Y. Liu, Z. Qin, M. Elkashlan, and Z, Ding, A. Nallanathan, L. Hanzo, "Nonorthogonal multiple access for 5G and beyond," Proc. IEEE, vol. 105, no. 12, pp. 2347-2381, Feb. 2017.

[14] M. Liu, J. Yang and G. Gui, "DSF-NOMA: UAV-assisted emergency communication technology in a heterogeneous internet of things," IEEE Internet Things J., vol. 6, no. 3, pp. 5508-5519, June 2019.

[15] Y. Yin, Y. Peng, M. Liu, J. Yang, and G. Gui, "Dynamic user groupingbased NOMA over Rayleigh fading channels," IEEE Access, vol. 7, no. 1, pp. 110964-110971, 2019.

[16] Z. Ding, P. Fan, G. K. Karagiannidis, R. Schober, and H. V. Poor, "NOMA assisted wireless caching: Strategies and performance analysis," IEEE Trans. Commun., vol. 66, no. 10, pp. 4854-4876, Oct. 2018.

[17] Z. Zhao, M. Xu, W. Xie, Z. Ding, and G. K. Karagiannidis, "Coverage performance of NOMA in wireless caching networks," IEEE Commun. Lett., vol. 22, no. 7, pp. 1458-1461, July 2018.

[18] Y. Fu, W. Wen, Z. Zhao, T. Q. S. Quek, S. Jin, and F. Zheng, "Dynamic power control for NOMA transmissions in wireless caching networks," IEEE Wireless Commun. Lett., vol. 8, no. 5, pp. 1485-1488, Oct. 2019.

[19] K. Shanmugam, N. Golrezaei, A. G. Dimakis, A. F. Molisch, and G. Caire, "FemtoCaching: Wireless content delivery through distributed caching helpers," IEEE Trans. Inf. Theory, vol. 59, no. 12, pp. 84028413, Dec. 2013. 